\title{
Interethnic Differences in Single and Haplotype Structures of Folylpolyglutamate Synthase and Gamma-glutamyl Hydrolase Variants and Their Influence on Disease Susceptibility to Acute Lymphoblastic Leukemia in the Indian Population: An Exploratory Study
}

\begin{abstract}
Aim: We aim to establish the genotype and haplotype frequencies of folylpolyglutamate synthase (FPGS rs10106 and rs1544105) and gamma-glutamyl hydrolase (GGH rs3758149 and rs11545078) variants in the South Indian population (SI) and to study the association of these variants with susceptibility to acute lymphoblastic leukemia (ALL). We also aim to compare the genotype and haplotype frequencies of studied variants with those of superpopulations from the 1000 Genomes Project collected in phase-3 and other published studies in the literature. Materials and Methods: A total of 220 unrelated healthy volunteers and 151 patients with ALL of both sexes were recruited for the study. Extracted DNA was subjected to genotyping by allelic discrimination using quantitative real-time-polymerase chain reaction. Genotype details of the studied variants in other ethnicities were obtained from 1000 genomes project Phase 3 data. Haploview

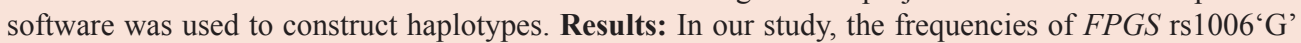
and rs 1544105 'A' alleles were found to be $37 \%$ and $37.2 \%$, respectively, and the frequencies of $G G H$ rs3758149'T' and $G G H$ rs11545078'T' alleles were found to be $29.8 \%$ and $16.7 \%$, respectively. Among the studied variants, FPGS rs $1544105^{\circ}$ AA' genotype carriers were found to be susceptible to the risk of ALL (odds ratio: $2.16 ; 95 \%$ confidence interval $[\mathrm{CI}]: 1.15-4.07 ; P=0.02$ ). Haplotype structures of FPGS and $G G H$ variants in SI population were significantly different from other ethnicities $(P<0.05)$, except the South Asian superpopulation. Conclusion: FPGS rs1544105'AA' genotype was found to influence the risk for ALL. Intra and interethnic differences exist in the distribution of studied variants. Therefore, the impact of each variant on the susceptibility and outcome of diseases may differ between populations.
\end{abstract}

Keywords: Antifolates, folate, folylpolyglutamate synthase, gamma-glutamyl hydrolase, haplotypes, polymorphism

\section{Introduction}

Acute lymphoblastic leukemia (ALL) is a hematologic malignancy characterized by the production of immature leukocytes. The estimated number of new cases of ALL in the United States in 2018 is 5960 and there are 1470 predicted deaths from ALL this year. ${ }^{[1]}$ In India, the lymphoid leukemia cases are expected to be 18,449 by the year 2020.[2] Both genetic and nongenetic factors play a role in ALL; however, despite many studies, the etiology of ALL is still poorly understood. Folate deficiency has been associated with the increased risk of some cancers, ${ }^{[3,4]}$ and lower folate levels were found to be associated with ALL in the Indian population. ${ }^{[5]}$ Folates

\footnotetext{
This is an open access journal, and articles are distributed under the terms of the Creative Commons Attribution-Non Commercial-ShareAlike 4.0 License, which allows others to remix, tweak, and build upon the work non-commercially, as long as appropriate credit is given and the new creations are licensed under the identical terms.
}

For reprints contact: reprints@medknow.com and antifolates are small molecules that are metabolized intracellularly into their more potent polyglutamate derivatives. Folylpolyglutamate synthase (FPGS) and gamma-glutamyl hydrolase $(G G H)$ are genes located on chromosomes 9 and 8 , respectively, that are essential for the intracellular accumulation of folate and antifolate polyglutamates. ${ }^{[6]}$ Mutations in FPGS and $G G H$ genes might affect the activity of these enzymes, altering intracellular levels of the context of the efficacy and safety of antifolate-based therapy. ${ }^{[10-12]}$ Genetic variants associated with disease among the

How to cite this article: Kodidela S, Pradhan SC, Dubashi B, Basu D. Interethnic differences in single and haplotype structures of folylpolyglutamate synthase and gamma-glutamyl hydrolase variants and their influence on disease susceptibility to acute lymphoblastic leukemia in the Indian population: An exploratory study. Indian J Med Paediatr Oncol 2018;39:331-8. polyglutamates [Table 1]..$^{[-9]}$ Variants in $F P G S$ and $G G H$ are also relevant in

\section{Sunitha Kodidela, Suresh Chandra Pradhan, Biswajit Dubashi' ${ }^{1}$, Debdatta Basu²}

Departments of Pharmacology, ${ }^{1}$ Medical Oncology and ${ }^{2}$ Pathology, Jawaharlal Institute of Postgraduate Medical Education and Research, Puducherry, India

Address for correspondence: Dr. Sunitha Kodidela, Jawaharlal Institute of Postgraduate Medical Education and Research, Puducherry - 605 006, India. E-mail: sunitha.kodidela@ gmail.com

Access this article online Website: www.ijmpo.org DOI: 10.4103/ijmpo.ijmpo_32_17 Quick Response Code:

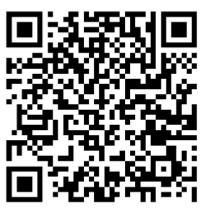




\begin{tabular}{lcclcll}
\hline \multicolumn{7}{c}{ Table 1: Genetic variants investigated in the study } \\
\hline Gene & rsid & $\begin{array}{c}\text { Nucleotide } \\
\text { change }\end{array}$ & $\begin{array}{l}\text { Type of } \\
\text { variant }\end{array}$ & $\begin{array}{c}\text { Chromosome } \\
\text { number: position }\end{array}$ & Effect on enzyme & $\begin{array}{c}\text { Consequences on } \\
\text { folate and MTX levels }\end{array}$ \\
\hline FPGS & rs1544105 & $2572 \mathrm{G}>\mathrm{A}$ & Intron & $9: 127800446$ & Decreased transcripts & Decreased \\
& rs10106 & $1944 \mathrm{~A}>\mathrm{G}$ & 3'UTR & $9: 127813796$ & Not known & - \\
\multirow{2}{*}{$G G H$} & rs3758149 & $-401 \mathrm{C}>\mathrm{T}$ & 5' UTR & $8: 63039169$ & Increased expression & Decreased \\
& rs11545078 & $452 \mathrm{C}>\mathrm{T}$ & Missense & $8: 63026205$ & Decreased activity & Increased $^{[9]}$ \\
\hline
\end{tabular}

UTR - Untranslated region; MTX - Methotrexate; FPGS - Folylpolyglutamate synthase; GGH - Gamma-glutamyl hydrolase

populations of other countries may not be associated with those in India $^{[13,14]}$ because Indians are genetically diverse and may differ from other populations..$^{[15-17]}$ To date, very few studies are available regarding the influence of FPGS and $G G H$ variants and their haplotypes on the risk of ALL in the global populace. Therefore, we aimed to establish the frequency of FPGS and GGH variants in healthy volunteers to provide a normative frequency which can be used to compare with those of patients with cancer risk.

\section{Materials and Methods}

The present study was approved by the JIPMER Institute's Ethics Committee (IEC; Number: JIP/IEC/SC/2/2012/28). Written informed consent was obtained from all study participants, and in the case of children, consent was obtained from their legally accepted representatives.

\section{Study population}

A case-control study consisting of 220 unrelated healthy volunteers (controls) and 151 patients (cases) with ALL of either sex was conducted. All the participants were residing in South India for at least three consecutive generations and spoke one of the Dravidian languages (Tamil, Telugu, Malayalam, or Kannada) as their mother tongue. The mean ages ( \pm standard deviation) of the cases and controls were $15.5( \pm 10.5)$ and $24.5( \pm 4.8)$ years, respectively. There were $99(65.6 \%)$ males and $52(34.4 \%)$ females in the patient group and $118(53.6 \%)$ males and $102(46.4 \%)$ females in the control group. We could not recruit age-matched healthy children due to difficulty in obtaining consent from patients' parents/legal guardians that resulted in the difference in mean age between cases and controls. Details of sample collection, DNA extraction, and quantification have been described previously. ${ }^{[18]}$ FPGS (rs10106 and rs1544105) and $G G H$ (rs11545078 and rs3758149) TaqMan assays were procured, to detect considered variants, from Applied Biosystems (Foster City, CA; USA). Genotyping was done by allelic discrimination using real-time polymerase chain reaction (Applied Biosystems-7300) according to the manufacturer's instructions. Genotyping was done in duplicates in $30 \%$ of the randomly selected samples and were found to be in 100\% concordance. Genotype details of the studied SNPs in other ethnic populations were obtained from the 1000 Genomes Project, phase-3, which include five major populations: Africans (AFR), Americans (AMR), East Asians (EAS), Europeans (EUR), and South Asians (SAS). We have also considered subpopulations of SAS such as Gujarati Indians from Houston (GIH), Punjabis from Lahore, Pakistan (PJL), Bengalis from Bangladesh (BEB), Sri Lankan Tamils from the UK (STU), and Indian Telugu from the UK (ITU) for the comparison of genotype frequencies.

\section{Construction of haplotypes and linkage disequilibrium}

To construct haplotype blocks and to obtain their corresponding frequencies, the genotype data of two loci per gene (FPGS, rs10106 and rs1544105 and GGH, rs3758149 and rs11545078) were used. Details of the variants are mentioned in Table 1.

A total of 218 and 211 samples of healthy volunteers were used for haplotype analysis of $G G H$ and $F P G S$ variants, respectively.

Haploview software v4.2 (Broad, Cambridge, MA, USA) ${ }^{[19]}$ was used to estimate the pairwise Linkage Disequilibrium (LD) pattern and haplotype frequencies. All markers/SNPs with minor allele frequencies $<0.05$ were excluded, and the minimum haplotype frequency was set at $1 \%$. Strong LD between a pair of markers is indicated by D' values from 0.7 to 1 , whereas moderate $\mathrm{LD}$ is indicated by $\mathrm{D}^{\text {' values }}$ from 0.2 to 0.7 and $D^{\prime}$ values from 0 to 0.2 indicate no linkage disequilibrium.

\section{Statistical analysis}

The observed genotype frequencies were tested for Hardy-Weinberg equilibrium (HWE) using the Chi-squared test. Fisher's exact test was used to test the differences in genotypes between ALL patients and healthy volunteers (controls), and odds ratios with $95 \%$ confidence interval were obtained. Comparison between genotype and allele frequencies of South Indians (SIs) with the 1000 Genomes Project data was made using the Chi-squared test. GraphPad InStat 3 (GraphPad Software Inc., San Diego, CA, USA) and SPSS software (version 16, SPSS Inc.; Chicago, IL, USA) were used for statistical analysis. The threshold for statistical significance was set at $P<0.05$.

\section{Results}

Comparison of genotype distribution of $F P G S$ and $G G H$ variants between patients with acute lymphoblastic leukemia and healthy individuals

The observed genotype frequencies of FPGS and $G G H$ variants in healthy individuals and patients with ALL 
were found to be in HWE $(P>0.05)$. Among the studied variants, FPGS rs1544105'AA' genotype carriers were found to be at risk of developing ALL [Table 2].

Comparison of haplotype structures of studied FPGS and $G G H$ variants between cases and controls

Haplotype structures (HS) of FPGS and $G G H$ variants were compared between cases and controls and were not found to be significantly different. There was however a trend observed with the GGH HS3 haplotype (carrying the variant allele ' $\mathrm{T}$ ' of both GGH-401 and 452) towards the protection against risk of ALL [Table 3], but it was not statistically significant $(P=0.06)$.

Comparison of frequency of studied variants in South Indian population with data from the 1000 Genomes Project and other studies

Allele frequencies of FPGS and $G G H$ variants in our healthy volunteers were compared with those of five superpopulations found in the 1000 Genomes Project phase 3-data and with other studies. Both FPGS rs10106 ' $G$ ' and rs1544105'A' alleles in the SI population (37\%) were significantly lower when compared to AFR, AMR, EAS, PJL, and Thai populations, ${ }^{[20]}$ but were similar to EUR and subpopulations of SAS (except PJL) [Table 4]. ${ }^{[21-29]}$

The frequency of the FPGS rs10106' $G$ ' allele in the SI population was also lower when compared to Puerto Rican, ${ }^{[21]}$ Dutch, ${ }^{[22]}$ and Singapore Chinese populations, ${ }^{[29]}$ whereas rs $1544105^{\prime} \mathrm{A}$ ' allele frequency was lower when compared to a Chinese population $(65.9 \%) .{ }^{[11]}$ There was also a significant difference in the distribution of genotype and allele frequencies of $G G H$ variants (GGH-401 (rs3758149) and $452 \quad \mathrm{C}>\mathrm{T} \quad(\mathrm{rs} 11545078)$ ) between the SI population and other ethnicities, except for subpopulations of SAS such as BEB, GIH, ITU, PJL, and STU [Table 4]. Frequency of the GGH-401'T' allele in the SI population $(29.8 \%)$ was significantly lower when compared to West Indians $(61 \%),{ }^{[28]}$ but it was similar to EUR, North Indian, ${ }^{[23]}$ and Thai

Table 2: Distribution of genotypes and allele frequencies of folylpolyglutamate synthase (rs10106 and rs1544105) and gamma-glutamyl hydrolase (rs3758149 and rs11545078) polymorphisms in patients with acute lymphoblastic leukemia and normal healthy individuals

\begin{tabular}{|c|c|c|c|c|}
\hline Genotypes and Alleles & Patients with ALL & Healthy volunteers & $P$ value & OR $(95 \%$ CI) \\
\hline FPGS $1944 \mathrm{~A}>\mathrm{G}(\mathrm{rs} 10106)$ & $N=145 ; n(\%)$ & $N=212 ; n(\%)$ & & \\
\hline AA & $49(33.8)$ & $82(38.7)$ & & 1.00 (reference) \\
\hline AG & $70(48.3)$ & $103(48.57)$ & 0.63 & $1.13(0.71-1.81)$ \\
\hline GG & $26(17.9)$ & $27(12.6)$ & 0.18 & $1.61(0.84-3.07)$ \\
\hline A & $168(57.9)$ & $267(63)$ & & 1.00 (reference) \\
\hline G & $122(42.1)$ & $157(37)$ & 0.81 & $1.2(0.91-1.67)$ \\
\hline FPGS $2572 \mathrm{G}>\mathrm{A}(\mathrm{rs} 1544105)$ & $N=149 ; n(\%)$ & $N=219 ; n(\%)$ & & \\
\hline \multicolumn{5}{|l|}{ Genetic models } \\
\hline \multicolumn{5}{|l|}{ Codominant model } \\
\hline GG & $44(29.5)$ & $83(37.9)$ & & 1.00 (reference) \\
\hline GA & $74(49.7)$ & $109(49.8)$ & 0.34 & $1.16(0.86-1.57)$ \\
\hline AA & $31(20.8)$ & $27(12.3)$ & $0.02 *$ & $2.16(1.15-4.07)$ \\
\hline \multirow[t]{2}{*}{ Recessive model GG + GA versus AA } & $118(79.2)$ & $192(87.7)$ & & 1.00 (reference) \\
\hline & $31(20.8)$ & $27(12.3)$ & $0.04 *$ & $1.40(1.06-1.85)$ \\
\hline G & $162(54.4)$ & $275(62.8)$ & & 1.00 (reference) \\
\hline A & $136(45.6)$ & $163(37.2)$ & $0.02 *$ & $1.41(1.05-1.91)$ \\
\hline$G G H-401 \mathrm{C}>\mathrm{T}(\mathrm{rs} 3758149)$ & $N=151 ; n(\%)$ & $N=220 ; n(\%)$ & & \\
\hline $\mathrm{CC}$ & $74(49.0)$ & $108(49.1)$ & & \\
\hline $\mathrm{CT}$ & $67(44.4)$ & $93(42.3)$ & 0.82 & $1.00(0.79-1.32)$ \\
\hline $\mathrm{TT}$ & $10(6.6)$ & $19(8.6)$ & 0.68 & $0.84(0.49-1.44)$ \\
\hline $\mathrm{C}$ & $215(71.2)$ & $309(70.2)$ & & 1.00 (reference) \\
\hline $\mathrm{T}$ & $87(28.8)$ & $131(29.8)$ & 0.80 & $0.97(0.80-1.17)$ \\
\hline$G G H 452 \mathrm{C}>\mathrm{T}(\mathrm{rs} 11545078)$ & $N=151 ; n(\%)$ & $N=218 ; n(\%)$ & & \\
\hline $\mathrm{CC}$ & $116(76.8)$ & $151(69.3)$ & & 1.00 (reference) \\
\hline $\mathrm{CT}$ & $34(22.5)$ & $61(28)$ & 0.41 & $0.39(0.06-2.50)$ \\
\hline $\mathrm{TT}$ & $1(0.7)$ & $6(2.7)$ & 0.24 & $0.32(0.05-2.03)$ \\
\hline $\mathrm{C}$ & $266(88.0)$ & $363(83.25)$ & & 1.00 (reference) \\
\hline $\mathrm{T}$ & $36(12)$ & $73(16.74)$ & 0.07 & $0.78(0.58-1.03)$ \\
\hline
\end{tabular}

$* P<0.05$. OR - Odds ratio; CI - Confidence interval; $N$ - Total number of patients considered to study the respective genetic variant; $n(\%)$ - Number of patients possessing respective particular genotype; ALL - Acute lymphoblastic leukemia 


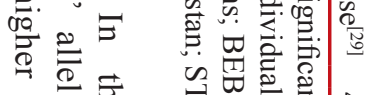

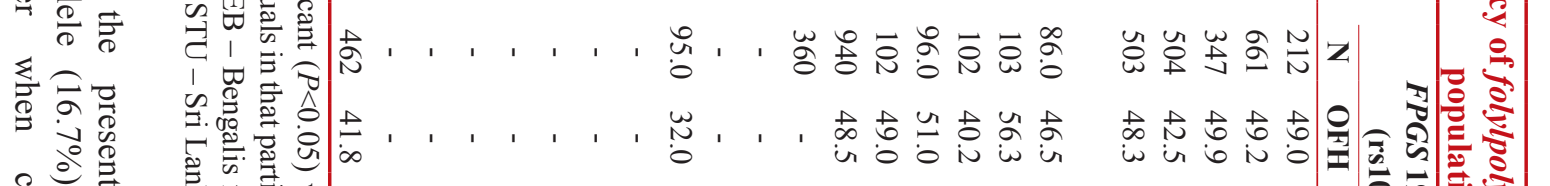

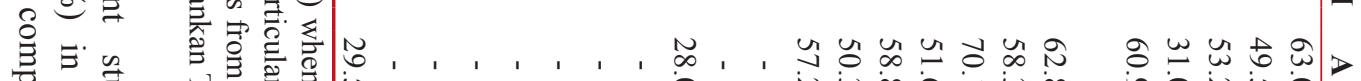
2 ○ $\overrightarrow{\mathrm{E}}$

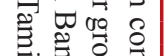

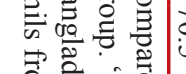

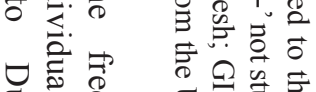

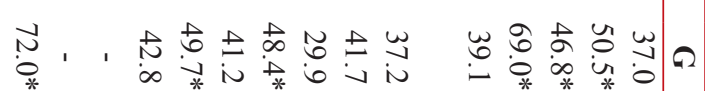

' के की त्र

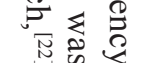

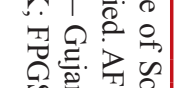

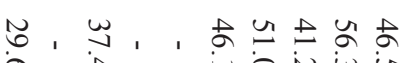

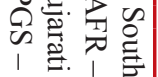

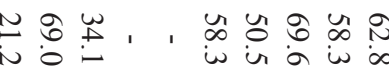
국. 뭉

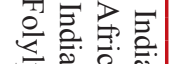
플. 药

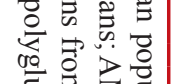

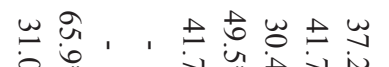

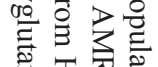

Z

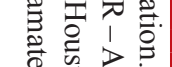

$\rightarrow 7$

龸害.

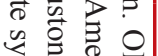

$\underset{N}{W} \stackrel{0}{-}$ 言恣敢 羟 용

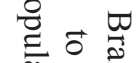

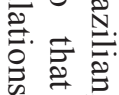

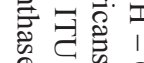

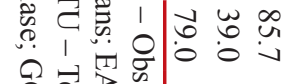

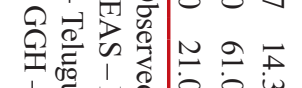

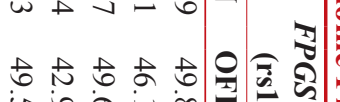

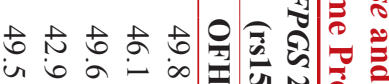

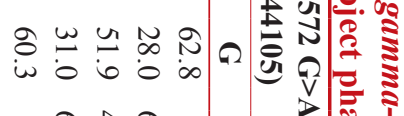

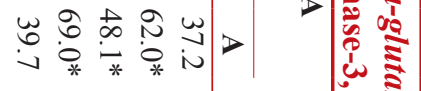
资

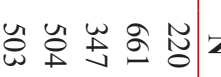

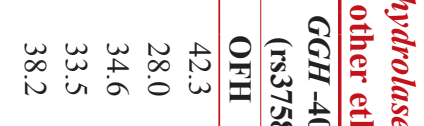

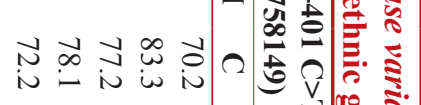
莺言芯

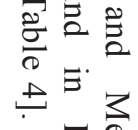
可

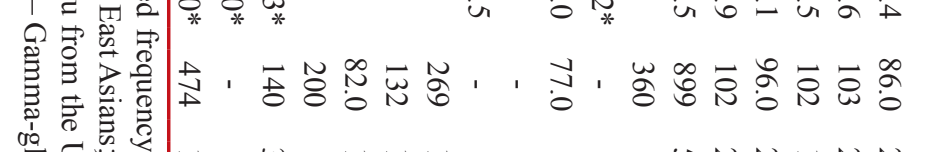

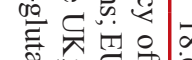

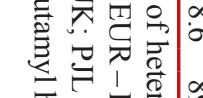

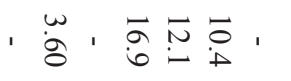

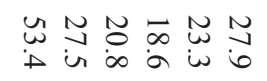

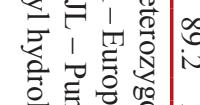

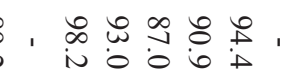

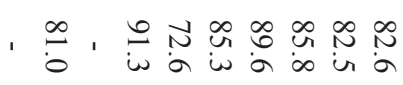

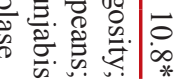

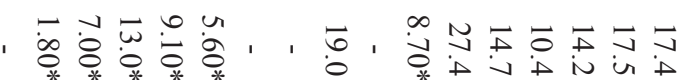

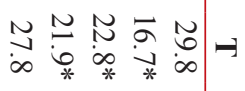

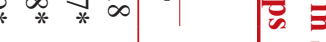

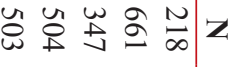
*****

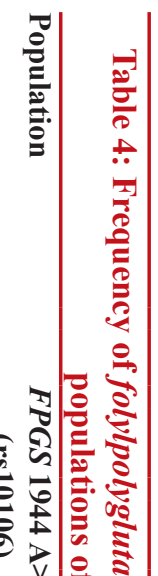

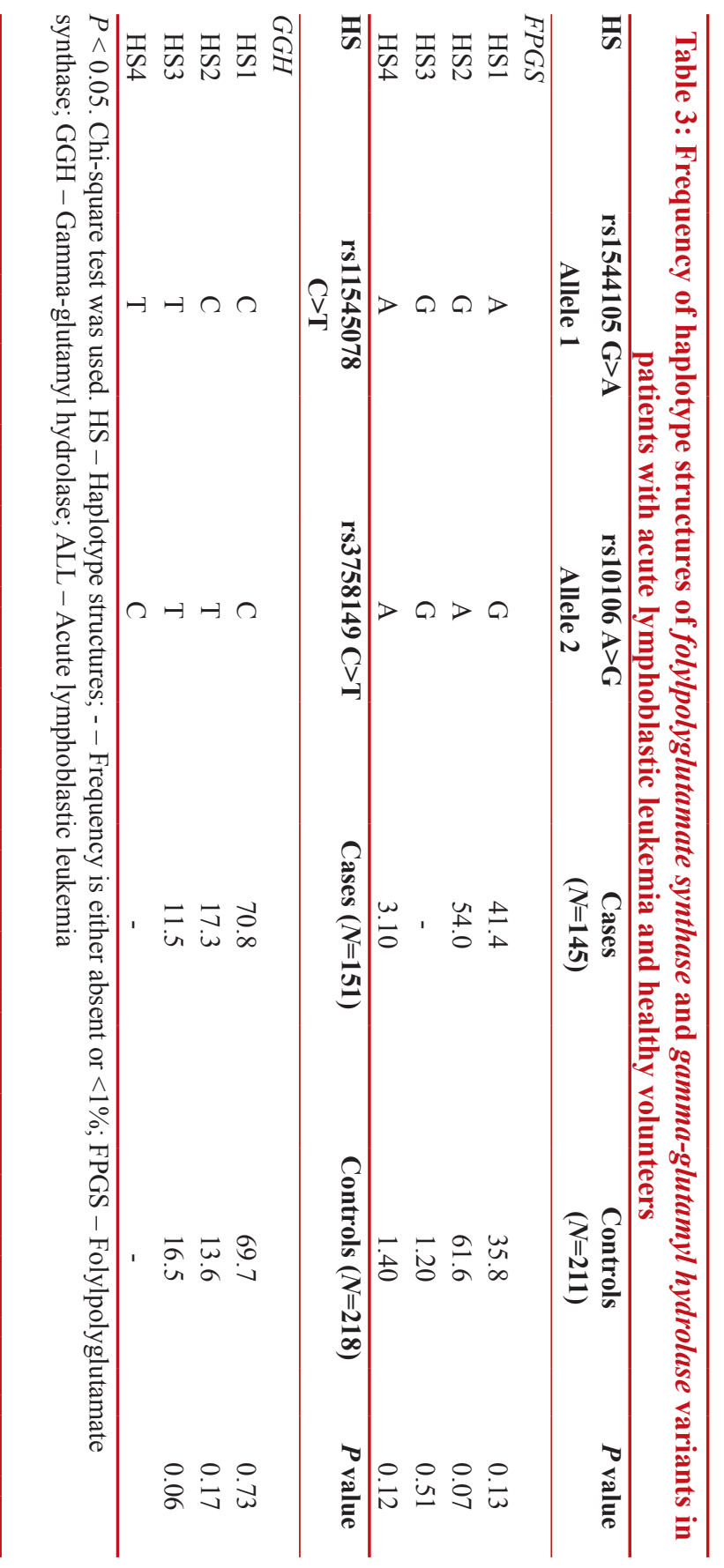




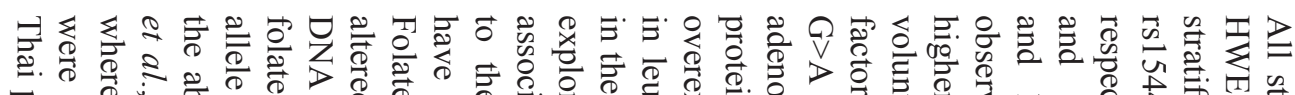

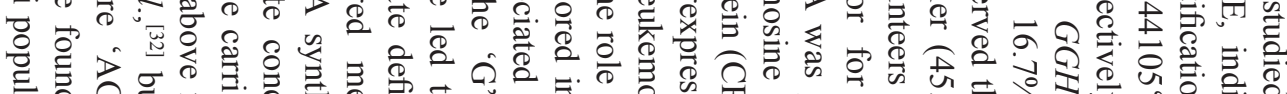

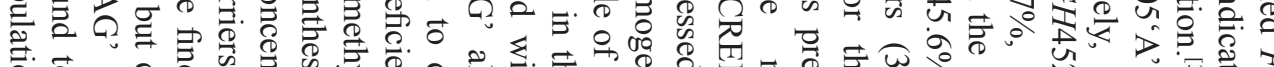

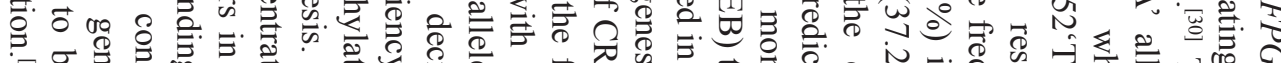
To 我

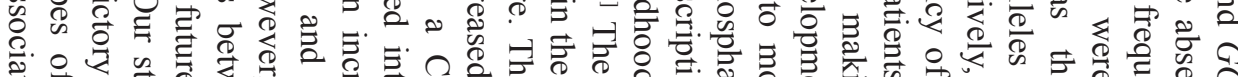

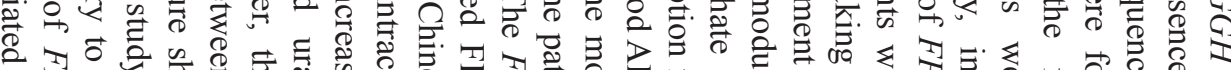

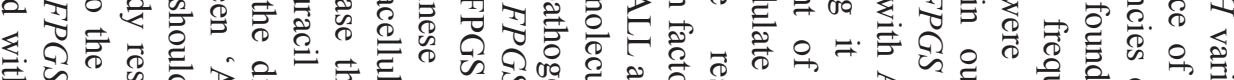

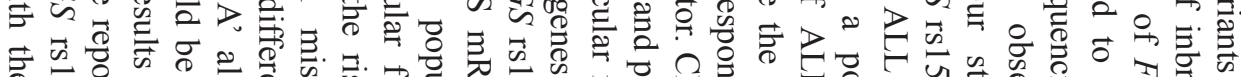

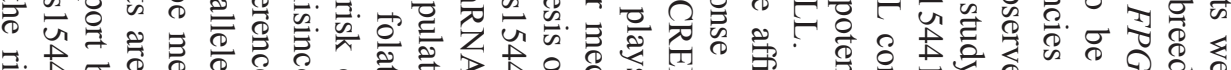

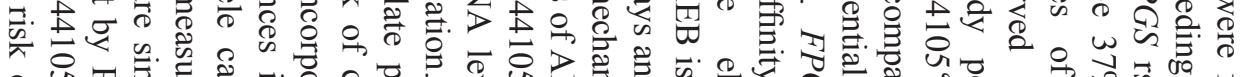

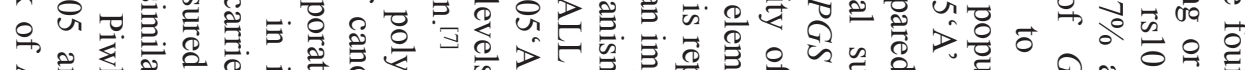

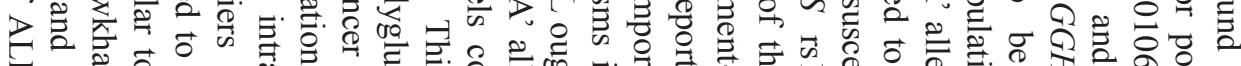

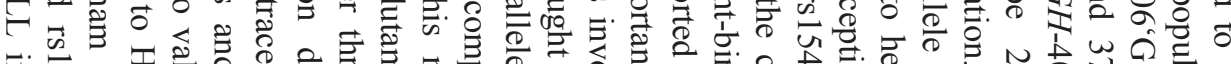
E.

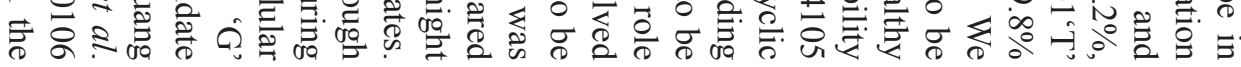

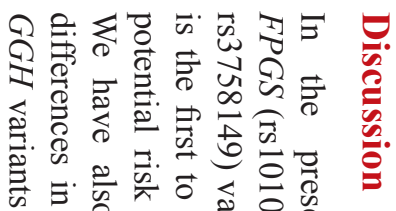

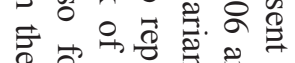

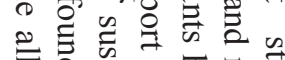

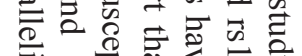

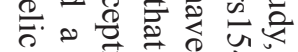

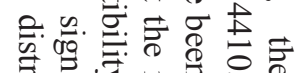

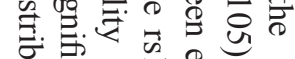

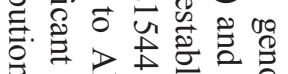
F. 뉸

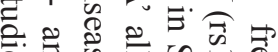

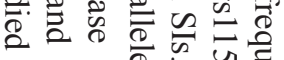

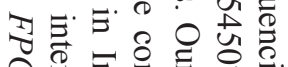

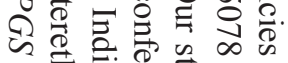

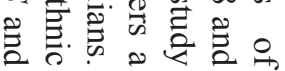

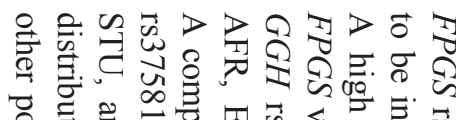

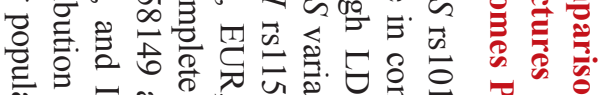

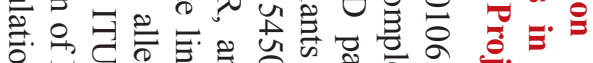

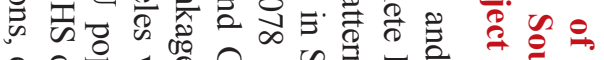

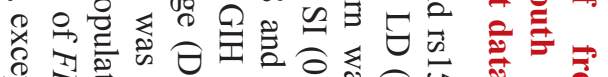
음

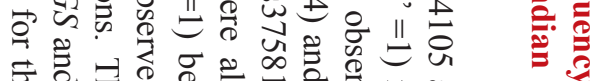

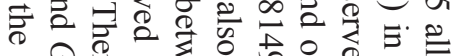

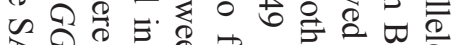

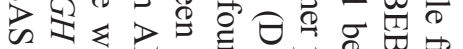

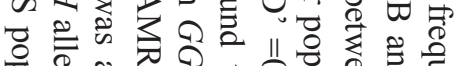

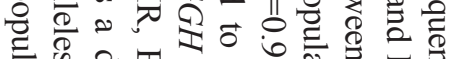

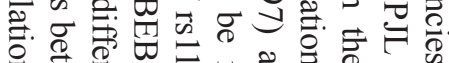

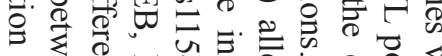
冚 $\frac{\sigma}{0} \Omega$

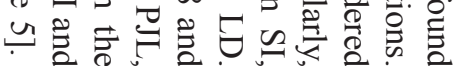

Table 5: Comparison of haplotype frequencies of folylpolyglutamate synthase and gamma-glutamyl hydrolase variants in South Indian population with the superpopulations of 1000 Genomes Project phase-3

\begin{tabular}{|c|c|c|c|c|c|c|c|c|c|c|c|c|}
\hline$\overline{\mathrm{HS}}$ & Allele 1 & Allele 2 & $\begin{array}{c}\text { Frequency } \\
\text { in SI (\%) } \\
(n=211)\end{array}$ & $\begin{array}{c}\text { Frequency } \\
\text { in AFR }(\%) \\
(n=661)\end{array}$ & $\begin{array}{c}\text { Frequency } \\
\text { in AMR } \\
(\%)(n=347)\end{array}$ & $\begin{array}{c}\text { Frequency } \\
\text { in EAS }(\%) \\
(n=504)\end{array}$ & $\begin{array}{c}\text { Frequency } \\
\text { in EUR }(\%) \\
(n=503)\end{array}$ & $\begin{array}{c}\text { Frequency } \\
\text { in BEB }(\%) \\
(n=86)\end{array}$ & $\begin{array}{c}\text { Frequency } \\
\text { in GIH (\%) } \\
(n=103)\end{array}$ & $\begin{array}{c}\text { Frequency } \\
\text { in ITU (\%) } \\
(n=102)\end{array}$ & $\begin{array}{c}\text { Frequency } \\
\text { in PJL }(\%) \\
(n=96)\end{array}$ & $\begin{array}{c}\text { Frequency } \\
\text { in STU (\%) } \\
(n=102) \\
\end{array}$ \\
\hline$\overline{F P G S}$ & rs1544105 G>A & rs10106 $\mathrm{A}>\mathrm{G}$ & & & & & & & & & & \\
\hline HS1 & A & G & 35.8 & $48.6^{*}$ & $45.6^{*}$ & $68 *$ & 38.1 & 37.2 & 41.3 & 29.4 & $48.4^{*}$ & 40.7 \\
\hline HS3 & G & G & 1.20 & 1.80 & 1.20 & 1.00 & 1.00 & - & - & - & - & - \\
\hline HS4 & A & A & 1.40 & $13.3 *$ & 2.50 & 1.00 & 1.60 & - & - & 1.00 & 1.00 & 1.00 \\
\hline$G G H$ & rs $11545078 \mathrm{C}>\mathrm{T}$ & rs $3758149 \mathrm{C}>\mathrm{T}$ & & & & & & & & & & \\
\hline HS1 & $\mathrm{C}$ & $\mathrm{C}$ & 69.7 & $83.0^{*}$ & $77.2^{*}$ & $78.1^{*}$ & 72.0 & 68.6 & 69.8 & 74.5 & 72.9 & 70.1 \\
\hline HS4 & $\mathrm{T}$ & $\mathrm{C}$ & 3.00 & 3.00 & - & - & - & - & 5.00 & - & - & - \\
\hline
\end{tabular}

*The values are significant $(P<0.05)$ when compared to those of South Indian population (SI). -: Indicate either absent or $<1 \%$. FPGS - Folylpolyglutamate synthase; GGH - Gamma-glutamyl hydrolase; AFR - Africans; AMR - Americans; EAS - East Asians; EUR - Europeans; SAS - South Asians; BEB - Bengalis from Bangladesh; GIH - Gujarati Indians from Houston; 
In the present study, the GGH452' $\mathrm{T}$ ' allele was not significantly associated with the risk of ALL [Table 2], and our results are in accordance with the previous studies conducted on Mexican $^{[12]}$ and Chinese populations. ${ }^{[25]}$

Furthermore, in the present study, the $G G H-401 \mathrm{C}>\mathrm{T}$ polymorphism was also not associated with the susceptibility to ALL. Our study results are similar to the findings by Koomdee et al. in a Thai population, ${ }^{[10]}$ but not in line with a study done on Mexican population where the $-401^{\prime} \mathrm{T}$ ' allele was associated with the risk of $\operatorname{ALL}(P=0.001) \cdot{ }^{[12]}$

These contradictory results could be due to differences in the frequency distribution of alleles ( $G G H-401^{\text {' }} \mathrm{T}$ ' allele in SI [29.8\%] vs. Thai population [23.5\%], $P=0.2$ and SI vs. Mexicans [14.3\%], $P<0.05$ ) [Table 5], and also the involvement of other enzymes in folate metabolism and differences in gene-environment interactions. Therefore, the effect of the $G G H-401 \mathrm{C}>\mathrm{T}$ polymorphism on the risk of ALL ought to be further studied along with other variants in the genes encoding folate-metabolizing enzymes. Comparison of HS of $G G H$ and FPGS variants between healthy volunteers and patients with ALL did not show a significant difference [Table 2].

Observation of genotype distribution of studied variants in other ethnicities revealed that FPGS rs10106'A' allele frequency was highest in ITU $(70.1 \%)$ followed by SI populations $(63 \%)$, and the ' $G$ ' allele was found at a higher frequency in the Thai population $(72 \%)^{[20]}$ followed by EAS $(69 \%)$. A significant difference existed in the distribution of FPGS variant alleles between SI and other populations from the 1000 Genomes Project, except Europeans and SAS [Table 5]. The allelic frequencies of FPGS variants in the SI population showed greatest similarity to genetically closer populations such as SAS, except the PJL population. BEB and GIH populations had a high occurrence of the $G G H-401^{\prime}$ ' $\mathrm{T}$ ' allele $(31.4 \%)$ and the $G G H 452^{\prime} \mathrm{T}$ ' allele $(17.5 \%)$, respectively. In a Mexican population, the frequencies of both $G G H-401^{\prime} \mathrm{C}$ ' $(85.7 \%)$ and $452^{\circ} \mathrm{C}$ ' $(98.2 \%)$ alleles were found to be higher compared to the present study. ${ }^{[12]} G G H-401^{\text {' }} \mathrm{T}$ ' allele frequency in the SI population significantly differed from the frequency in a West Indian population. ${ }^{[28]}$ The significant differences in FPGS variants between SI and PJL populations and $G G H$ variants between SI and West Indians suggest that populations with similar geographical background may not be considered together because they may possess significant differences in their genetic loci.

Haplotype analysis revealed a high LD pattern between studied FPGS (rs10106 and rs1544105) variants (D' $>0.95$ ). FPGS HS2 was the most frequent haplotype in SI, followed by HS1, HS4, and HS3, in descending order. HS3 frequency was found to be $<3 \%$ in all populations. The frequency of HS4 carrying variant allele of rs1544105' $\mathrm{A}^{\prime}$ was high in AFR (13.3\%) whereas it was either absent or occurred at $<3 \%$ frequency in other populations. Therefore, the influence of these polymorphisms on disease susceptibility and drug response might vary in AFR, relative to other populations. There was a significant difference in the distribution of the HS of FPGS alleles between SI and other populations. $G G H$ rs11545078 and rs3758149 alleles were also found to be in strong LD in SI population. HS1 of $G G H$ was the predominant haplotype in all populations, followed by HS3 and HS2 in SI, BEB, $\mathrm{GIH}$, and ITU. HS2 is the second most frequent haplotype in AMR, EAS, EUR, PJL, and STU, followed by HS3. HS4 was found at very low frequencies in SI, AFR, and GIH populations and was absent in other populations. The HS3 haplotype (16.5\%) of $G G H$ carrying variant allele was higher in SI population compared to other populations, except BEB and GIH. Significant differences in HS between SI and other populations could be due to differences in allele frequencies, suggesting interethnic variations in the susceptibility to disease and response to treatment. Limitation of our study might be a lack of data on folate levels that could have strengthened our findings. Therefore, folate levels need to be measured at the time of disease diagnosis in the future. Other variants in the genes encoding folate-metabolizing enzymes also need to be explored, to find a reliable biomarker for susceptibility to ALL disease.

Clinical relevance of $F P G S$ and $G G H$ variants in acute lymphoblastic leukemia

FPGS and GGH enzymes are involved in both folate and antifolate metabolism. Therefore, changes in the activities of these enzymes due to genetic variants may the influence the levels of antifolates and there by affect the treatment response also. Patients with FPGS rs $1544105^{~ ' C C}$ ' genotype had lower relapse-free survival $(P=0.01)$ and event-free survival $(P=0.04)$, but did not develop MTX toxicity. ${ }^{[7]}$ Higher FPGS activity was associated with accumulation of long-chain MTXPGs and better overall survival in patients with ALL. ${ }^{[33]}$ The GGH452'TT+CT' genotype was associated with increased risk of hepatotoxicity and mucositis, but not hematological toxicity, in a Chinese population. ${ }^{[25]}$ In European children, the $G G H 452^{\text {' }} \mathrm{T}$ ' allele was associated with thrombocytopenia, but neither $G G H$ polymorphisms nor haplotypes were associated with MTX response and survival. ${ }^{[34]} G G H-401 \mathrm{C}>\mathrm{T}$ and 'TT' genotype carriers were at increased risk of developing leukopenia and thrombocytopenia after high-dose methotrexate in a Thai population. ${ }^{[10]}$ In a Mexican population, the $G G H-401 \mathrm{C}>\mathrm{T}$ polymorphism was found to increase the risk of relapse significantly whereas $G G H 452 \mathrm{C}>\mathrm{T}$ polymorphism did not affect ALL outcome. ${ }^{[12]}$ In Chinese patients, a higher serum MTX concentration/dose ratio and a higher concentration of MTX above the therapeutic threshold $(>40 \mu \mathrm{M})$ were observed in $G G H$ rs3758149'CT' or 'TT' genotype carriers when compared to ' $\mathrm{CC}$ ' genotype carriers after high-dose MTX therapy. However, FPGS polymorphism was not 
associated with serum MTX levels. ${ }^{[11]}$ The above-observed differences in clinical outcome of ALL between various ethnicities could partly be explained by the differences in the distribution of $G G H$ and FPGS variant alleles. Therefore, the impact of each SNP on the susceptibility and outcome of diseases might vary among different populations.

\section{Conclusion}

In our study, the FPGS rs $1544105^{\circ} \mathrm{AA}$ ' genotype was found to be associated with the susceptibility to ALL in SI population. Genotype and haplotype distributions of FPGS (rs10106 and rs1544105) and GGH (rs3758149 and rs11545078) variants in the SI population significantly differed from those of other ethnicities. Our data emphasized that each ethnicity has unique allele frequencies of studied FPGS and GGH variants. Thus, knowledge of genotype frequency distribution within a population can be useful to tailor drug therapy by optimizing drug doses and identifying potential risk groups which may develop toxicity.

\section{Compliance with ethical standards}

All procedures performed in our study were in accordance with the ethical standards of the institutional and/or national research committee and with the 1964 Helsinki declaration and its later amendments or comparable ethical standards.

\section{Acknowledgments}

We would like to thank Jawaharlal Institute of Postgraduate Medical Education and Research, Puducherry, India, for providing an intramural fund to conduct the study.

\section{Financial support and sponsorship}

Nil.

\section{Conflicts of interest}

There are no conflicts of interest.

\section{References}

1. Siegel RL, Miller KD, Jemal A.Cancer statistics, 2016.CA Cancer J Clin 2016;66:7-30.

2. Takiar R, Nadayil D, Nandakumar A. Projections of number of cancer cases in India (2010-2020) by cancer groups. Asian Pac J Cancer Prev 2010;11:1045-9.

3. Duthie SJ. Folic acid deficiency and cancer: Mechanisms of DNA instability. Br Med Bull 1999;55:578-92.

4. Zhou X, Meng Y. Association between serum folate level and cervical cancer: A meta-analysis. Arch Gynecol Obstet 2016;293:871-7.

5. Sadananda Adiga MN, Chandy S, Ramaswamy G, Appaji L, Aruna Kumari BS, Krishnamoorthy L, et al. Association between plasma homocysteine and riboflavin status in acute lymphoblastic leukemia in children. Indian J Clin Biochem 2009;24:257-61.

6. Longo GS, Gorlick R, Tong WP, Lin S, Steinherz P, Bertino JR, et al. Gamma-glutamyl hydrolase and folylpolyglutamate synthetase activities predict polyglutamylation of methotrexate in acute leukemias. Oncol Res 1997;9:259-63.
7. Liu SG, Gao C, Zhang RD, Jiao Y, Cui L, Li WJ, et al. FPGS rs1544105 polymorphism is associated with treatment outcome in pediatric B-cell precursor acute lymphoblastic leukemia. Cancer Cell Int 2013;13:107.

8. Chave KJ, Ryan TJ, Chmura SE, Galivan J. Identification of single nucleotide polymorphisms in the human gamma-glutamyl hydrolase gene and characterization of promoter polymorphisms. Gene 2003;319:167-75.

9. Cheng Q, Wu B, Kager L, Panetta JC, Zheng J, Pui CH, et al. A substrate specific functional polymorphism of human gamma-glutamyl hydrolase alters catalytic activity and methotrexate polyglutamate accumulation in acute lymphoblastic leukaemia cells. Pharmacogenetics 2004;14:557-67.

10. Koomdee N, Hongeng S, Apibal S, Pakakasama S. Association between polymorphisms of dihydrofolate reductase and gamma glutamyl hydrolase genes and toxicity of high dose methotrexate in children with acute lymphoblastic leukemia. Asian Pac J Cancer Prev 2012;13:3461-4.

11. Wang SM, Sun LL, Zeng WX, Wu WS, Zhang GL. Influence of genetic polymorphisms of FPGS, GGH, and MTHFR on serum methotrexate levels in chinese children with acute lymphoblastic leukemia. Cancer Chemother Pharmacol 2014;74:283-9.

12. Organista-Nava J, Gómez-Gómez Y, Saavedra-Herrera MV, Rivera-Ramírez AB, Terán-Porcayo MA, Alarcón-Romero Ldel C, et al. Polymorphisms of the gamma-glutamyl hydrolase gene and risk of relapse to acute lymphoblastic leukemia in Mexico. Leuk Res 2010;34:728-32.

13. Rani DS, Carlus SJ, Poongothai J, Jyothi A, Pavani K, Gupta NJ, et al. CAG repeat variation in the mtDNA polymerase gamma is not associated with oligoasthenozoospermia. Int $\mathrm{J}$ Androl 2009;32:647-55.

14. Mehrotra S, Oommen J, Mishra A, Sudharshan M, Tiwary P, Jamieson SE, et al. No evidence for association between SLC11A1 and visceral leishmaniasis in India. BMC Med Genet 2011;12:71.

15. Indian Genome Variation Consortium. Genetic landscape of the people of India: A canvas for disease gene exploration. J Genet 2008;87:3-20.

16. Reich D, Thangaraj K, Patterson N, Price AL, Singh L. Reconstructing Indian population history. Nature 2009;461:489-94.

17. Tamang R, Singh L, Thangaraj K. Complex genetic origin of Indian populations and its implications. J Biosci 2012;37:911-9.

18. Kodidela S, Pradhan SC, Dubashi B, Basu D. Influence of dihydrofolate reductase gene polymorphisms rs408626 $(-317 \mathrm{~A}>\mathrm{G})$ and $\mathrm{rs} 442767(-680 \mathrm{C}>\mathrm{A})$ on the outcome of methotrexate-based maintenance therapy in South Indian patients with acute lymphoblastic leukemia. Eur J Clin Pharmacol 2015;71:1349-58.

19. Barrett JC, Fry B, Maller J, Daly MJ. Haploview: Analysis and visualization of $\mathrm{LD}$ and haplotype maps. Bioinformatics 2005;21:263-5.

20. Piwkham D, Siriboonpiputtana T, Beuten J, Pakakasama S, Gelfond JA, Paisooksantivatana K, et al. Mutation screening and association study of the folylpolyglutamate synthetase (FPGS) gene with susceptibility to childhood acute lymphoblastic leukemia. Asian Pac J Cancer Prev 2015;16:4727-32.

21. DeVos L, Chanson A, Liu Z, Ciappio ED, Parnell LD, Mason JB, et al. Associations between single nucleotide polymorphisms in folate uptake and metabolizing genes with blood folate, homocysteine, and DNA uracil concentrations. Am J Clin Nutr 2008;88:1149-58.

22. van der Straaten RJ, Wessels JA, de Vries-Bouwstra JK, Goekoop-Ruiterman YP, Allaart CF, Bogaartz J, et al. 
Exploratory analysis of four polymorphisms in human GGH and FPGS genes and their effect in methotrexate-treated rheumatoid arthritis patients. Pharmacogenomics 2007;8:141-50.

23. Sharma S, Das M, Kumar A, Marwaha V, Shankar S, Aneja R, et al. Interaction of genes from influx-metabolism-efflux pathway and their influence on methotrexate efficacy in rheumatoid arthritis patients among Indians. Pharmacogenet Genomics 2008;18:1041-9.

24. Hayashi H, Fujimaki C, Inoue K, Suzuki T, Itoh K. Genetic polymorphism of C452T (T127I) in human gamma-glutamyl hydrolase in a Japanese population. Biol Pharm Bull 2007;30:839-41.

25. Chen X, Wen F, Yue L, Li C. Genetic polymorphism of $\gamma$-glutamyl hydrolase in Chinese acute leukemia children and identification of a novel double nonsynonymous mutation. Pediatr Hematol Oncol 2012;29:303-12.

26. Zhang HH, Yue LJ, Chen XW, Zhao W, Hu CY, Zheng MM, et al. Analysis of a $452 \mathrm{C} / \mathrm{T}$ single nucleotide polymorphism in $\gamma$-glutamyl hydrolase gene in children with acute leukemia. Zhonghua Yi Xue Yi Chuan Xue Za Zhi 2012;29:352-5.

27. Chiabai MA, Lins TC, Pogue R, Pereira RW. Population analysis of pharmacogenetic polymorphisms related to acute lymphoblastic leukemia drug treatment. Dis Markers 2012;32:247-53.

28. Ghodke Y, Chopra A, Shintre P, Puranik A, Joshi K,
Patwardhan B, et al. Profiling single nucleotide polymorphisms (SNPs) across intracellular folate metabolic pathway in healthy Indians. Indian J Med Res 2011;133:274-9.

29. Oppeneer SJ, Ross JA, Koh WP, Yuan JM, Robien K. Genetic variation in folylpolyglutamate synthase and gamma-glutamyl hydrolase and plasma homocysteine levels in the Singapore Chinese Health Study. Mol Genet Metab 2012;105:73-8.

30. Wigginton JE, Cutler DJ, Abecasis GR. A note on exact tests of hardy-weinberg equilibrium. Am J Hum Genet 2005;76:887-93.

31. Pigazzi M, Ricotti E, Germano G, Faggian D, Aricò M, Basso G, et al. CAMP response element binding protein (CREB) overexpression CREB has been described as critical for leukemia progression. Haematologica 2007;92:1435-7.

32. Huang Z, Tong HF, Li Y, Qian JC, Wang JX, Wang Z, et al. Effect of the polymorphism of folylpolyglutamate synthetase on treatment of high-dose methotrexate in pediatric patients with acute lymphocytic leukemia. Med Sci Monit 2016;22:4967-73.

33. Wojtuszkiewicz A, Peters GJ, van Woerden NL, Dubbelman B, Escherich G, Schmiegelow K, et al. Methotrexate resistance in relation to treatment outcome in childhood acute lymphoblastic leukemia. J Hematol Oncol 2015;8:61.

34. Garcia-Bournissen F, Moghrabi A, Krajinovic M. Therapeutic responses in childhood acute lymphoblastic leukemia (ALL) and haplotypes of gamma glutamyl hydrolase (GGH) gene. Leuk Res 2007;31:1023-5. 